\title{
Exchange between deep donors in semiconductors: a quantum defect approach
}

\author{
W. $\mathrm{Wu}^{*}$ and A.J. Fishen $\dagger^{\dagger}$ \\ UCL Department of Physics and Astronomy and London Centre for Nanotechnology, \\ University College London, Gower Street, London WC1E 6BT
}

(Dated: November 4, 2018)

\begin{abstract}
Exchange interactions among defects in semiconductors are commonly treated within effectivemass theory using a scaled hydrogenic wave-function. However such a wave-function is only applicable to shallow impurities; here we present a simple but robust generalization to treat deep donors, in which we treat the long-range part of the wavefunction using the well established quantum defect theory, and include a model central-cell correction to fix the bound-state eigenvalue at the experimentally observed value. This allows us to compute the effect of binding energy on exchange interactions as a function of donor distance; this is a significant quantity given recent proposals to carry out quantum information processing using deep donors. As expected, exchange interactions are suppressed (or increased), compared to the hydrogenic case, by the greater localization (or delocalization) of the wavefunctions of deep donors (or 'super-shallow' donors with binding energy less then the hydrogenic value). The calculated results are compared with a simple scaling of the Heitler-London hydrogenic exchange; the scaled hydrogenic results give the correct order of magnitude but fail to reproduce quantitatively our calculations. We calculate the donor exchange in silicon including inter-valley interference terms for donor pairs along the $\{100\}$ direction, and also show the influence of the donor type on the distribution of nearest-neighbour exchange constants at different concentrations. Our methods can be used to compute the exchange interactions between two donor electrons with arbitrary binding energy.

PACS numbers: 03.67.Lx, 71.55.Cn
\end{abstract}

\section{INTRODUCTION}

Accurate estimations of exchange interactions among semiconductor defects are crucial in determining the magnetic properties of doped semiconductors near to a metal-insulator transition [1] and in assessing the potential of such defects for potential applications in quantum information processing [2, 3]. However, the long-range tails of defect wavefunctions make it difficult or impossible to treat these systems using fully ab initio techniques, so such calculations are generally performed within empirical models such as the well-established effective mass approximation [4, 5, 6]. The quantum-mechanical problem for a single defect is thereby reduced to that of a single electron moving in an effective medium determined by the effective mass and static permittivity of the host crystal; in the simplest form of the theory, the solution becomes that of a scaled hydrogen atom. The exchange between two such defects can then be obtained from well-established treatments of exchange in the hydrogen molecule, ranging from the simple Heitler-London model 7] to the more sophisticated approach of Herring and Flicker [8, 9] which exactly accounts for the two-electron correlations in the limit of large separations.

However, a number of complications arise when considering real defect systems. First, the minima of the conduction band may not be isotropic; this is so in the important case of silicon, where there are six degenerate min-

\footnotetext{
*Electronic address: wei.wu@ucl.ac.uk

${ }^{\dagger}$ Electronic address: andrew.fisher@ucl.ac.uk
}

ima lying along the $\{100\}$ directions in the Brillouin zone and each having a significant anisotropy $\left(m_{\perp}=0.98 m_{e}\right.$, $\left.m_{\|}=0.19 m_{e}\right)$, where $m_{e}$ is electron mass. This is commonly dealt with either by simply adopting an effective isotropic dispersion with an appropriate average effective mass (for example, $m^{*}=\left(m_{\|} m_{\perp}^{2}\right)^{1 / 3}$ ), or by making a variational ansatz for the wavefunction of the anisotropic system, such as that of Luttinger and Kohn [4, , 5, [6] .

Second, the existence of more than one minimum needs to be accounted for; this results in an effective-mass wavefunction of the form

$$
\psi(\vec{r})=\sum_{n} \alpha_{n} F_{n}(\vec{r}) \phi_{n \vec{k}_{0}}(\vec{r}),
$$

where the sum runs over the different minima, $F_{n}$ is a slowly-varying envelope function, and $\phi_{n \vec{k}_{0}}(\vec{r})=\exp \left(\vec{k}_{0}\right.$. $\vec{r}) u_{n \vec{k}_{0}}(\vec{r})$ is the Bloch function for the $n$th minimum. The coefficients $\alpha_{n}$ arise from the coupling of the different band minima by short-range (large-wavevector) components of the potential; for an isolated substitutional donor in a perfect crystal, they correspond to an irreducible representation of the $T_{d}$ point group. In this paper we concentrate on the ground state of defects in silicon, for which the terms in equation (11) correspond to the six $\{100\}$ directions and the appropriate representation is the identity representation:

$$
\alpha^{(0)}=\frac{1}{\sqrt{6}}(1,1,1,1,1,1) .
$$

The existence of these distinct parts to the wavefunction gives rise to interference terms in properties of defect pairs as a function of their separation. Such terms were 
originally considered in electron hopping and recombination rates [10, 11], and later included in exchange interactions [1, 12]. Other more recent calculations are discussed below. Note, however, that the expressions used in [1] to average the exchange over the different minima assume a particular physical origin for the exchange (namely the direct electron-electron Coulomb interaction term) which does not, in fact, dominate the problem. Although its magnitude has approximately the same asymptotic scaling as the full exchange, by itself the direct term would lead to a ferromagnetic spin-spin interaction. The observed interaction is always antiferromagnetic, as it must be for a two-electron system [14]).

Both the anisotropy and multiple-minima problems can be solved while remaining largely or entirely within the framework of effective-mass theory, and a number of recent papers have addressed the exchange between hydrogenic donors by this method. Much of the inspiration for this work came from the proposal [2] to use such exchange in shallow donors as a qubit-qubit coupling mechanism to drive entangling gates in quantum information processing. The importance of precise donor positioning in determining the value of the exchange was emphasized in [13], but this paper follows [1] in using only the (ferromagnetic) direct exchange interaction for the calculations. The full Heitler-London formula for the exchange was used in a following paper [15], where the contribution of strain to modifying the interaction was also discussed. These calculations were performed by neglecting the spatial variation of the periodic part of the Bloch functions $u_{n, \vec{k}_{0}}$ appearing in equation (11); this approximation was carefully examined in [16] and found to be very accurate, and this finding was confirmed by a subsequent calculation of the electronic structure of defect pairs in which the Bloch functions from an ab initio treatment of the host crystal were used [17]. The same paper also found that allowing the phases of the different Bloch function contributions to 'float' relative to one another in the case of the defect pair made small difference to the results.

The third difficulty is in many ways most serious: effective-mass theory predicts that all single-electron donors in a given host should have the same binding energy, independent of their chemical nature. This is far from the case: in silicon, for example, observed binding energies range from $31.2 \mathrm{meV}$ for $\mathrm{Li}$ and $45.6 \mathrm{meV}$ for $\mathrm{P}$ through to $71.0 \mathrm{meV}$ for $\mathrm{Bi}$ [18], whereas the calculated value using the Luttinger-Kohn variant of effective mass theory is $31.3 \mathrm{meV}$ [18]. It is very desirable to predict exchange in deeper donors by similarly simple methods: they are advantageous for quantum information processing [3] because they are less prone to ionization, and have much longer spin-lattice relaxation lifetimes [19] indeed, resonant Raman routes for spin relaxation are completely absent if the level spacing exceeds the maximum phonon energy of the host material $(64.5 \mathrm{meV}$ for $\mathrm{Si})$.

Treating deep donors requires some significant correc- tions to effective mass theory. Two common approaches involve making an explicit short-range correction to the Coulomb field of the impurity (the so-called 'central cell correction'), or altering the long-range solution in the Coulomb field so that it corresponds to the observed binding energy (the 'quantum defect' approach, which has its origins in atomic physics) [20, 21]. However, the effect of these modifications on the exchange interactions of the defects has remained largely unknown.

In this paper we show how the exchange in the most physically relevant range of separations can be computed between donors with arbitrary binding energies (including the important case of deep donors). We use a quantum-defect description, with a simple model potential to represent the central-cell correction; for reasons that are explained below, it is important to compute the exchange using a potential for which the wavefunction concerned is an eigenfunction. We first introduce the techniques that we use: the effective-mass model, quantum-defect corrections to it and the simple centralcell corrections, followed by the methods we use to calculate exchange. Then we present our results for defects of different binding energies.

\section{METHOD OF CALCULATION}

\section{A. Effective-mass theory}

The effective-mass equation [4, 5, [6] reads:

$$
\left[\epsilon_{n}\left(\vec{k}_{0}+\frac{1}{i} \nabla\right)+U\right] F_{n}=\epsilon F_{n} .
$$

where it is intended that the band energy $\epsilon_{n}$ be expanded around the band extremum $\vec{k}_{0}$ to second order-terms in $(1 / i) \nabla . F_{n}$ is the envelope function, in terms of which the true wavefunction is expanded using

$$
\psi=\sum_{n} \alpha_{n} F_{n}(\vec{r}) \phi_{n \vec{k}_{0}}(\vec{r}),
$$

where $F_{n}$ is a solution of the effective mass equation.

In the simplest theory, the effective mass tensor is replaced by a single averaged effective mass $m^{*}$, resulting in an effective isotropic equation for the envelope function, which is then independent of the index $n$ :

$$
\left[-\frac{\hbar^{2}}{2 m^{*}} \nabla^{2}-\frac{e^{2}}{\epsilon_{r} r}-\epsilon\right] F(\vec{r})=0,
$$

where $\epsilon_{r}$ is the relative permittivity of the host. In this paper we will follow other recent treatments (for example $[13,15])$ and work with this isotropic equation as our starting point. For silicon, $m^{*}=0.33 m_{e}$ and $\epsilon_{r}=11.7$; this leads to a set of scaled atomic units for the hydrogenic impurity problem (length $a_{0}^{*}=1.94 \mathrm{~nm}$, energy $\left.\mathrm{Ha}^{*}=0.062 \mathrm{eV}\right)$. 


\section{B. The quantum defect method}

The first component of our correction to effective-mass theory involves treating the wavefunction far from the impurity by the quantum defect method [21]. Even in the simplest isotropic approximation, the method was found to provide quantitative results giving good approximations to both the observed spectral dependence and magnitude of the photo-ionization cross section [20]. An essential feature of the quantum defect method is that good approximate wave functions valid in the region outside the impurity ion core can be determined using only a knowledge of the energy eigenvalues. Rather than attempting to solve it as an eigenvalue equation to determine the allowed spectrum of $\epsilon$, equation (5) is considered valid for large $r$ only and solved for the asymptotic form of the envelope functions $F(\vec{r})$ corresponding to the $\mathrm{em}$ pirical value of $\epsilon$. Therefore the method can deduce the long-range part of the donor wave functions associated with the observed energy levels, whether deep or shallow, provided that the dominant corrections to effective-mass theory are short-range (operate only near the defect). Of the several different terms believed to contribute to the shift in binding energy for deep donors (see, for example, [22]), only the electron-phonon interaction operates far from the defect and its contribution is believed to be small.

The most useful form of the far-field solution is a multiple of the well-known Whittaker function which is just a particular linear combination of two standard linearly independent confluent hypergeometric functions, the combination being determined by the boundary conditions at infinity. We consider the auxiliary radial function defined by $P(r) \equiv r R(r)$, and write

$$
P_{\nu, l}(r)=N_{\nu, l} W_{\nu, l+\frac{1}{2}}(2 r / \nu)
$$

where $W$ is a Whittaker function [23], $N$ is a normalization constant, and $\nu$ is the quantum defect parameter defined by

$$
\epsilon=\frac{-1}{2 \nu^{2}}
$$

If and only if the donor is hydrogenic (i.e. shallow), $\nu$ is an integer.

\section{Model central cell corrections}

For non-integer values of $\nu$, the radial function associated with the solution (6) diverges like $R(r)=P(r) / r \sim$ $\frac{1}{r}$ as $r \rightarrow 0$. (Nevertheless this divergence is integrable, so the functions have a well-defined normalization for all $\nu$ [21].) In order to find a solution to equation (5) that remains finite at the origin for a general given energy $\epsilon$, we have to correct the potential at short distances. One way to do this is to look for corrections based on the local physics of the impurity (for example incorporating correctly the transition from a screened to an unscreened nuclear potential [24, 25, 26], or including self-consistently the scattering effects of the impurity by means of a pseudoptential based on the microscopic physics [27]); another way is to correct the potential at small $r$ empirically solely in order to make the solution regular there at the experimentally observed energy eigenvalue. In this second case the potential will not correspond to the physics operating in the core region of the real defect, but it will produce the correct shift in binding energy. We adopt the second approach in this paper, and refer to the empirical correction as a model central-cell correction. We should expect that the two approaches would give similar results for the calculation of exchange interactions, since they are determined predominantly by the long-range behaviour of the wavefunction.

We can then write the Hamiltonian of a single defect center $A$ as

$$
\hat{H}_{A}=-\frac{1}{2} \nabla^{2}-\frac{1}{\left|\vec{r}-\vec{R}_{A}\right|}+\delta V\left(\vec{r}-\vec{R}_{A}\right),
$$

where the third term on the right hand side is model central cell correction, which is not unique. In this work we used two forms for $\delta V$ : a $\delta$-function shell or a squarewell potential.

\section{1. $\delta$ potential correction}

Let us choose $\delta V$ to be a potential "shell" at radius $r=a$, where $a$ is very small $(a \ll 1$ in scaled atomic units):

$$
V(r)=-\frac{1}{r}+\lambda \delta(r-a) .
$$

In the following discussion, we will only consider ssymmetry wave functions because they are the donor ground states. After matching the large- $r$ solution to a solution valid for small $r$ that is regular at the origin and obeys the cusp condition for a nucleus of charge $Z=1$ $\left(R^{\prime}(0)=-R(0)\right)$, we find the solution is

$$
R(r)=\left\{\begin{array}{ll}
R(a) \frac{1-r}{1-a} & : \quad r \leq a \\
P_{\nu, 0}(r) / r & : \quad r>a
\end{array}\right\},
$$

where $P_{\nu, 0}(r)$ is the long-range solution for s-states defined by equation (6). Figure (1) shows the wave function for deep and shallow donors, both with the central cell correction and without it. Note how the diverging tails of the Whittaker function are cut off for $r<a$.

\section{Square-well potential correction}

An alternative matching is to replace the Coulomb potential by a square well, of depth $V_{0}$, for $r<a$ :

$$
V(\vec{r})=\left\{\begin{array}{rll}
V_{0} & : & r \leq a \\
-\frac{1}{r} & : & r>a
\end{array}\right\}
$$


This model correction gives us:

- $E>V_{0}$

$$
P(r)=\left\{\begin{array}{rll}
P_{\nu, 0}(a) \frac{\sin k r}{\sin k a} & : & r \leq a \\
P_{\nu, 0}(r) & : & r>a
\end{array}\right\}
$$

- $E<V_{0}$

$$
P(r)=\left\{\begin{array}{rll}
P_{\nu}(a) \frac{\sinh k r}{\sinh k a} & : & r \leq a \\
P_{\nu}(r) & : & r>a
\end{array}\right\} .
$$

In Figure (1) we compare the wave functions for both deep and shallow donors, with both $\delta$-function squarewell potential central cell corrections, and without any central-cell correction. Note that in both cases the diverging tails of the wave function are cut off for $r<a$.

\section{Exchange calculations}

Within effective-mass theory, the exchange between two hydrogenic donors as a function of distance maps to the exchange between two hydrogen atoms as a function of bond length. This problem has been treated using different methods since the 1920s.

\section{The Heitler-London model}

The Heitler-London model [7] simply evaluated the difference between the expectation values of the Hamiltonian in two two-electron wave functions, one a singlet $\left({ }^{1} \Sigma_{g}\right)$ and one a triplet $\left({ }^{3} \Sigma_{u}\right)$, both constructed from the 1s ground states $\phi_{a}(\vec{r})$ and $\phi_{b}(\vec{r})$ of the single atoms. This approach neglects any contributions from other atomic states, and hence also contributions to the correlation arising from the polarization of one atom in the field of the other. The energy difference can be written as

$$
\begin{aligned}
J \equiv E_{t} & -E_{s}=\frac{2}{1-\widetilde{s}^{4}}\left[2 j \widetilde{s}^{2}+j^{\prime} \widetilde{s}^{2}-2 k \widetilde{s}-k^{\prime}\right], \\
\widetilde{s} & \equiv \int \phi_{a}(1) \phi_{b}(1)[1] ; \\
j & =\int \phi_{a}(1)\left[-\frac{1}{r_{1 b}}\right] \phi_{a}(1)[1] ; \\
k & =\int \phi_{a}(1)\left[-\frac{1}{r_{1 b}}\right] \phi_{b}(1)[1] ; \\
j^{\prime} & \equiv \int \phi_{a}^{2}(1) \phi_{b}^{2}(2) \frac{1}{r_{12}}[1][2] ; \\
k^{\prime} & \equiv \int \phi_{a}(1) \phi_{b}(1) \phi_{a}(2) \phi_{b}(2) \frac{1}{r_{12}}[1][2] .
\end{aligned}
$$

All these quantities are positive; the one-electron contributions involving $j$ and $k$ yield a net antiferromagnetic coupling (positive $J$ in our sign convention), while the

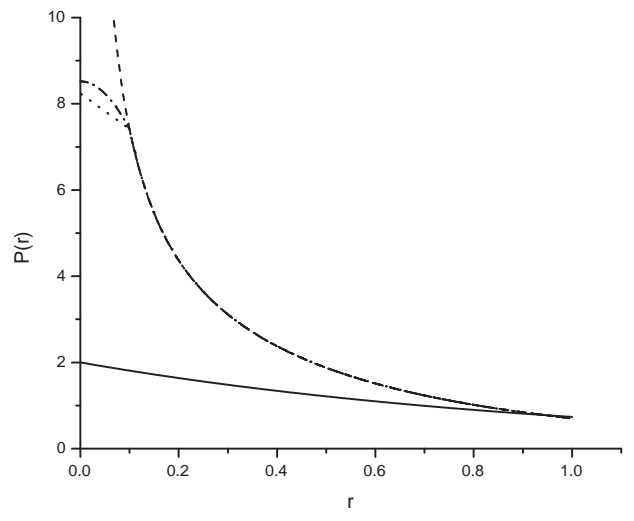

(a)

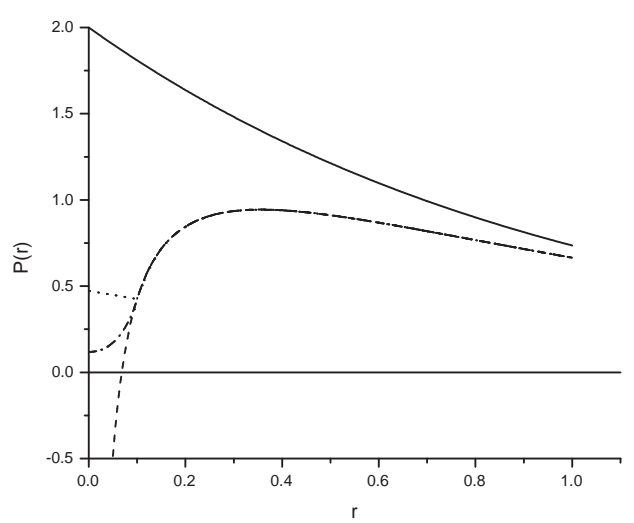

(b)

FIG. 1: Comparison of the wavefunctions generated by the $\delta$ potential and square-well potential central-cell corrections. (a) Deep donor, $\nu=0.7$; (b) super-shallow donor, $\nu=1.1$. In each case, the dotted curve shows $P_{\nu, 0}^{\delta}(r) / r$ with a $\delta$-potential central cell correction applied at $a=0.1$, the dashed-dotted curve is $P_{\nu, 0}^{s w}(r) / r$ with a square-well potential central cell correction applied with $a=0.1$; dashed curve: uncorrected Whittaker function $P_{\nu, 0} / r$ plotted for comparison; solid curve: radial part of a true $1 s$ Hydrogenic state $(\nu=1)$. Notice how the diverging tail of the Whittaker function is cut off for $r<a$ by both central cell corrections; the form of the curves for $r<a$ depends on the details of the potential applied.

two-electron terms $j^{\prime}$ and $k^{\prime}$ are net ferromagnetic. However, the neglect of correlation results in an unphysical logarithmic divergence in $k^{\prime}$ at large internuclear separations $R$, causing the overall $J$ to become negative (ferromagnetic), in violation of the theorem proved by Heisenberg [14] showing that the spatial ground-state of a two-electron system is always even under exchange of the particle positions, and hence is a spin singlet.

We note that, in order to write $J$ in the form of equation (14), it is essential that $\phi_{a}$ and $\phi_{b}$ be exact eigenstates of the single-atom problem with energy $E_{0}=-1 / 2$. This allows one to replace the off-diagonal 
matrix elements of the kinetic energy operator by

$$
\int \phi_{a}(1)\left[-\frac{1}{2} \nabla_{1}^{2}\right] \phi_{b}(1)[1]=\int \phi_{a}(1)\left[E_{0}+\frac{1}{r_{1 b}}\right] \phi_{b}(1)[1] .
$$

We refer to this as the 'Koiller method', since it is used in 13,15$]$.

If (20) is not obeyed (i.e. if $\phi_{a}$ and $\phi_{b}$ are not exact eigenfunctions of the single-donor problem), an alternative expression must be used:

$$
\begin{aligned}
\left\langle\psi_{\mp}|\hat{H}| \psi_{\mp}\right\rangle= & \frac{1}{1 \mp S^{2}}\left[2\left\langle\phi_{a}(1)\left|\left(-\frac{1}{2} \nabla^{2}\right)\right| \phi_{a}(1)\right\rangle+2\left\langle\phi_{a}(1)\left|\left(-\frac{1}{r_{1 A}}\right)\right| \phi_{a}(1)\right\rangle\right. \\
& +2\left\langle\phi_{a}(1)\left|\left(-\frac{1}{r_{1 B}}\right)\right| \phi_{a}(1)\right\rangle \mp 2 S\left(\left\langle\phi_{a}(1)\left|\left(-\frac{1}{2} \nabla^{2}\right)\right| \phi_{b}(1)\right\rangle+2\left\langle\phi_{a}(1)\left|\left(-\frac{1}{r_{1 A}}\right)\right| \phi_{b}(1)\right\rangle\right) \\
& \left.+\int d[1] d[2] \frac{\phi_{a}^{2}(1) \phi_{b}^{2}(2)}{r_{12}} \mp \int d[1] d[2] \frac{\phi_{a}(1) \phi_{b}(1) \phi_{a}(2) \phi_{b}(2)}{r_{12}}\right]+\frac{1}{R} \\
J= & \left\langle\psi_{-}|\hat{H}| \psi_{-}\right\rangle-\left\langle\psi_{+}|H| \psi_{+}\right\rangle .
\end{aligned}
$$

We refer to this as the 'exact Heitler-London' method below.

In either case, when using a central-cell correction of the type discussed in $₫ \mathrm{IIC}$ it is important to include the central-cell terms in the appropriate matrix elements of the single-particle potential, i.e. in the calculation of the quantities $j$ and $k$ via equations (16) and (17), and in the one-electron terms in (21).

\section{Other trial wavefunctions}

Other trial wavefunctions have been proposed for the hydrogenic case in an attempt to give some account of electron-electron correlation and remove the unphysical sign change in $J$. Kolos eq al. 29] used trial wave functions in elliptic-coordinates similar to those proposed by [28] to perform a variational calculation of singlet and triplet energies, obtaining the exchange splitting from the energy difference. The numerical results are compared with the Heitler-London values in Figure (2).

\section{The Herring-Flicker asymptotic form}

A different approach was pursued by Herring [8] and by Gor'kov and Pitaevskii [30], who pointed out that the exchange could be written in terms of a hyper-surface integral as

$$
J=\frac{1}{2} \int_{S} \mathbf{d} \mathbf{S}\left[\left(P \Phi_{1}\right) \nabla \Phi_{1}-\Phi_{1} \nabla\left(P \Phi_{1}\right)\right]+\mathrm{O}\left(\mathrm{e}^{-4 R}\right),
$$

where

$$
\Phi_{1}=\frac{1}{\sqrt{2}}\left(\phi_{g}+\phi_{u}\right)
$$

is a spatial wavefunction that is not properly antisymmetrized and has electron 1 localized on atom $a$ and electron 2 on atom $b$, while (assuming the nuclear separation is in the $z$ direction) the hypersurface $S$ is defined by the condition $z_{1}=z_{2}$.

Herring and Flicker [9] showed that this formula could be evaluated exactly in the limit $R \rightarrow \infty$, giving an asymptotic form for the exchange of

$$
\begin{aligned}
J_{\mathrm{H}-\mathrm{F}} & =E_{\text {triplet }}-E_{\text {singlet }} \\
& =1.642 R^{5 / 2} e^{-2 R}+O\left(R^{2} e^{-2 R}\right) .
\end{aligned}
$$

No unphysical sign change occurs in the Herring-Flicker asymptotic form.

We compare the Heitler-London, Kolos and HerringFlicker results for values of $R$ up to $7 a_{0}$ (using a logarithmic scale) in Figure 2(a); we also give a comparison for larger values of $R$ (up to 20a $)$ in Figure 2(b). Note that all three calculations agree quite well up to $R=7 a_{0}$ (the maximum radius for which Kolos' numerical results were calculated), and that the Heitler-London and HerringFlicker forms are also close for $R \leq 20 a_{0}$. We therefore 
expect that the Heitler-London approximation is a reasonable one in this distance range (the unphysical sign change to a ferromagnetic interaction does not occur until $R=49.5 a_{0}$ ). For comparison, Figure 2(b) also shows the variation of the quantity $\mu_{0} g^{2} \mu_{B}^{2} / 4 \pi r^{3}$, which determines the magnitude of the magnetic dipolar interaction; we see that the dipolar interaction starts to dominate over exchange for hydrogenic defects when $R \geq 12 a_{0}^{*}$.

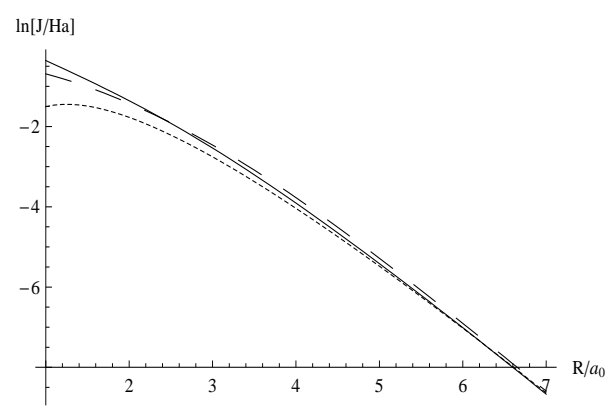

(a)

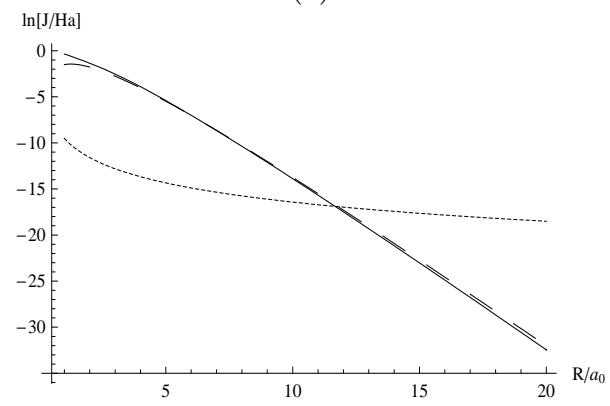

(b)

FIG. 2: The single-envelope exchange splitting $J$ for hydrogenic donors calculated by three different methods as a function of inter-nuclei distance $R$ and shown on a logarithmic scale. (a) Heitler-London exchange (solid curve), Herring-Flicker asymptotic (dotted curve) and Kolos' numerical calculation (dashed curve) for $R \leq 7 a_{0}$; (b) HeitlerLondon exchange (solid curve), Herring-Flicker asymptotic (dotted curve) and magnitude of magnetic dipolar interaction for $R \leq 20 a_{0}$. Notice that Heitler-London model agrees closely with the Herring-Flicker asymptotic form, and that from around $R=12 a_{0}$ the dipole-dipole interaction is larger than the exchange interaction. exchange formula (14) should be replaced [15] by

$$
\begin{aligned}
J(\vec{R})= & \sum_{\nu, \mu}\left[\sum_{\vec{K}, \vec{K}^{\prime}}\left|c_{\vec{K}}^{\nu}\right|^{2}\left|c_{\vec{K}^{\prime}}^{\mu}\right|^{2} e^{i\left(\vec{K}-\vec{K}^{\prime}\right) \cdot \vec{R}}\right] \\
& \times\left|\alpha_{\nu}\right|^{2}\left|\alpha_{\mu}\right|^{2} J_{\nu \mu}(\vec{R}) \cos \left(\vec{k}_{\nu}-\vec{k}_{\mu}\right) \cdot \vec{R}
\end{aligned}
$$

where the pair of donors are at $\vec{R}_{A}=0, \vec{R}_{B}=\vec{R}$ and $R>>a_{0}^{*}$ (effective Bohr radius). The second sum (in square brackets) in equation (26) refers to the reciprocallattice expansion of the periodic Bloch functions, $u_{\nu}(\vec{r})=$ $\sum_{\vec{K}} c_{\vec{K}}^{\nu} e^{i \vec{K} \cdot \vec{r}}$, and $\vec{k}_{\nu}, \vec{k}_{\mu}$ are band minima points. The full expression for $J_{\nu \mu}$ is given in the Appendix of [15]; in the isotropic effective-mass approximation where the envelope functions $F_{n}(\vec{r})$ are the same for each minimum, and assuming that rapidly oscillating terms in the integrals (proportional to $e^{i\left(\vec{k}^{(i)}-\vec{k}^{(j)}\right) \cdot \vec{r}}$, where $r$ is one of the integrated variables) are negligible, $J_{\mu \nu}$ can be replaced by the exchange $J_{w}$ computed using the radial functions derived from the Whittaker functions. The final expression for the exchange is then

$$
\begin{aligned}
J(R)= & \sum_{\nu, \mu}\left[\sum_{\vec{K}, \vec{K}^{\prime}}\left|c_{\vec{K}}^{\nu}\right|^{2}\left|c_{\vec{K}^{\prime}}^{\mu}\right|^{2} e^{i\left(\vec{K}-\vec{K}^{\prime}\right) \cdot \vec{R}}\right] \\
& \times\left|\alpha_{\nu}\right|^{2}\left|\alpha_{\mu}\right|^{2} J_{w}(R) \cos \left(\vec{k}_{\nu}-\vec{k}_{\mu}\right) \cdot \vec{R} .
\end{aligned}
$$

\section{E. Fitting Whittaker function with $1 s$ Gaussian}

Once modified by our model central cell correction, Whittaker functions are valid solutions for the singleimpurity problem for the whole range of $r$. To evaluate the integrals appearing in $J_{w}(R)$, we expand these solutions as a sum of Gaussians and use the analytical formulae in [31]. We write

$$
\begin{array}{r}
\frac{P_{\nu, 0}(r)}{r} \simeq R_{\nu}^{G}(r)=\sum_{n} A_{n} G_{n}\left(r, B_{n}\right) \\
G_{n}\left(r, B_{n}\right)=e^{-B_{n} r^{2}} .
\end{array}
$$

In all the calculations presented here, we use ten $1 s$ Gaussian-type orbitals to fit each Whittaker function.

In Figure (3) we show comparisons of such fits with the original wave functions. We see that we obtain a very good fit over the physically interesting distance range $R \leq 12 a_{0}^{*}$.

\section{Inter-valley effects}

The calculations presented so far give the exchange for molecular hydrogen. When we consider impurity states in a semiconductor we must remember that the full wavefunction is of the form (4). Assuming that the intervalley couplings are mainly determined by single-impurity physics (so equation (2) still holds), the Heitler-London

\section{RESULTS}

\section{A. Choice of parameters}

We select parameters appropriate for donors in silicon, as described previously: the appropriate effective mass is $m^{*}=\left(m_{\perp}^{2} * m_{\|}\right)^{\frac{1}{3}}=0.33 m_{e}$ and the relative permittivity $\epsilon_{r}=11.7$, leading to an effective Bohr radius 


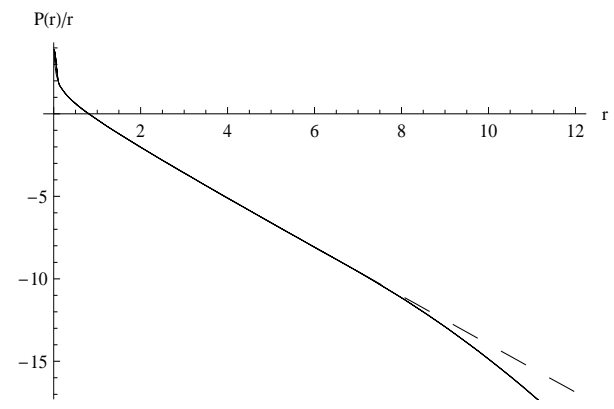

(a)

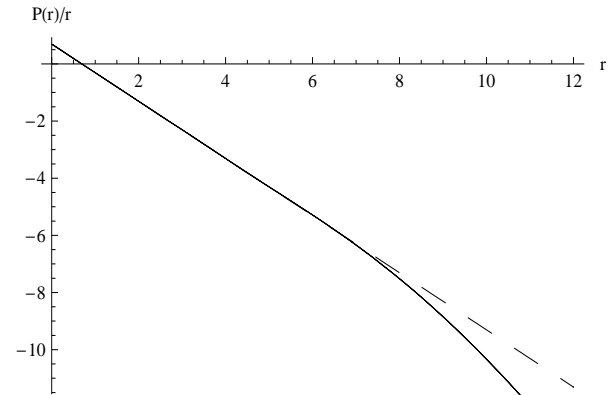

(b)

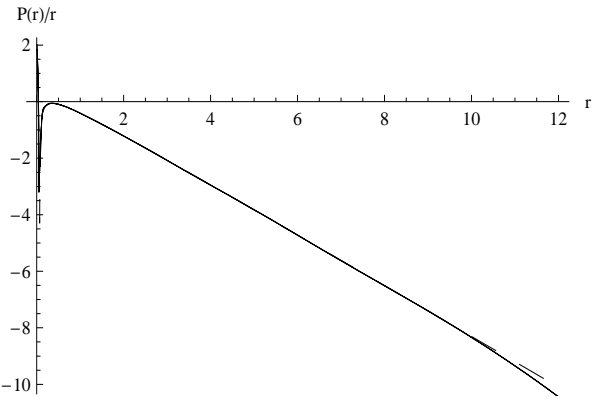

(c)

FIG. 3: Gaussian fits of Whittaker functions for (a) typical deep donor $(\nu=0.7)$, (b) typical hydrogenic shallow donor electron $(\nu=1.0)$ and (c) 'super-shallow' donor $(\nu=1.1)$. In each case the solid curve is the Gaussian fit and the dashed curve is the Whittaker function $P(r) / r$.

$a_{0}^{*}=0.053 \mathrm{~nm} \frac{\epsilon_{r} m^{*}}{m_{e}}=1.94 \mathrm{~nm}$ and an effective Hartree $\mathrm{Ha}^{*}=27.2 \mathrm{eV}\left(a_{0} / a_{0}^{*}\right)=62 \mathrm{meV}$.

We take as a typical deep donor the case of $\mathrm{Bi}: \mathrm{Si}$, for which the binding energy is about $71 \mathrm{meV}$ [18] or approximately $1.15 \mathrm{Ha}^{*}$; according to formula (7), we therefore adopt $\nu=0.7$ as a typical value for a deep donor, as used in Figure (11). For comparison and to show that our method is also capable of dealing with the case $\nu>1$, we also show results for the case $\nu=1.1$, corresponding to a hypothetical 'super-shallow' donor whose binding energy is less than the effective-mass value. (This is of less practical interest, as the shallowest known donor, Li:Si, has $\nu$ almost exactly equal to 1.) In all case where a model central-cell correction (of either type) is used, the radius is set to $a=0.01 a_{0}^{*}$.

The results presented here cover the distance range from $a_{0}^{*}$ to $12 a_{0}^{*}$. This range includes the lengthscales of greatest interest in practice, both for studies of the metal-insulator transition and for applications in quantum information processing, since it runs from the typical nearest-neighbour separation at the highest attainable densities below the metal-insulator transition (at a donor density $n_{D}=4 \times 10^{18} \mathrm{~cm}^{-3}$, the mean interdonor spacing is $3.5 \mathrm{~nm}$ or $1.8 a_{0}^{*}$ and approximately $90 \%$ of nearest-neighbour separations are greater than $a_{0}^{*}$ ) up to separations where the dipole-dipole interaction starts to dominate over exchange (see Figure 2).

\section{B. Benchmark: hydrogenic donors, $\nu=1.0$}

First, we perform a benchmark calculation in which we compute the Heitler-London exchange interaction between two pure hydrogenic donors (i.e. $\nu=1$ ) by two different methods: first using the standard evaluation of the integrals from the exact Coulomb wavefunctions, and second using our Gaussian fit. The results are shown in Figure (4); the excellent agreement between the dotted line $\mathbf{A}$ (exact Coulomb wavefunctions) and the solid line C (Gaussian fit) shows that the errors introduced by the Gaussian fit are negligible. There is however some deviation at large distances $\left(R \geq 8 a_{0}^{*}\right)$ between the solid curve $\mathbf{C}$ (exact Heitler-London result) and the dashed curve B (Koiller method); this arises because our Gaussian fit is not an exact eigenfunction of the one-centre problem and culminates in an unphysical cusp in curve B (corresponding to a sign-change in the exchange) at $R \approx 11 a_{0}$.

\section{Deep donors, $\nu=0.7$}

Now we know that the errors arising from our Gaussian fits are likely to be small in the region of interest, we can move on to calculate our main result: the exchange coupling between two deep donors. The results for $J_{w}$, calculated from a single envelope function, are shown in Figure (5). Notice that the six curves shown have very similar behaviours over most of the distance range. The three curves calculated using the 'exact Heitler-London method' and the different model central-cell corrections $(\mathbf{A}, \mathbf{C}, \mathbf{E})$ are very close to one another, but in this case there are some significant deviations among the curves calculated using 'Koiller's method'(B, D, F), both from the exact Heitler-London results and among one another. These deviations arise because the left side and right sides in equation (20) are not exactly equal: in the cases of curves $\mathbf{D}$ and $\mathbf{F}$, where central-cell corrections are included, this discrepancy arises from errors in the Gaussian fit, whereas in the case of curve $\mathbf{B}$ (no central-cell correction) there is an additional error because the Whittaker function is not a true eigenfunction of the potential. This introduces a larger error and leads to an (unphysical) cusp corresponding to a sign-change in the predicted exchange at $R \approx 11 a_{0}^{*}$ 


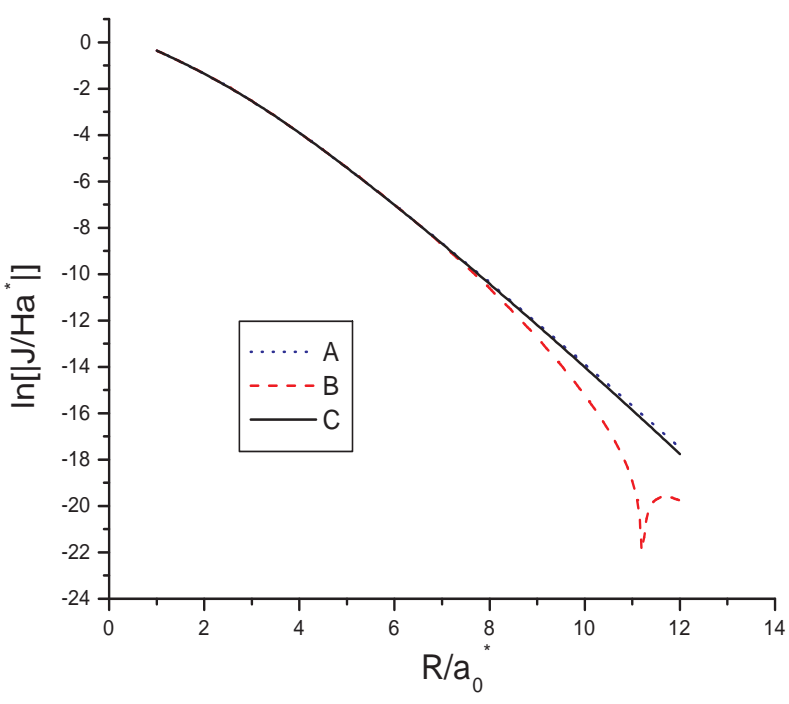

FIG. 4: (Colour online.) The exchange coupling between hydrogenic donors as a function of inter-nuclear distance $R$. Blue dotted curve (A): exchange calculated directly from Hydrogenic 1s Coulomb wave function; red dashed curve (B): exchange from Koiller's method based on $R_{1.0}^{G}$; black solid curve $(\mathbf{C})$ : exact Heitler-London method from $R_{1.0}^{G}$.

Comparing the computed exchange with the results for hydrogenic states (Figure 4), we see that the dominant effect is the change in exponential decay constant reflecting the change in the exponent of the radial wavefunctions at large distances. It is therefore natural to ask whether a good approximation to exchange in deep donors can be obtained simply by re-scaling the results from the Heitler-London approach for a hydrogenic donor, making the replacement $R \rightarrow R / \nu$. This comparison is made in Figure 6(a); we see that the scaled interactions have qualitatively the right behaviour and the correct order of magnitude, but do not match the details of our calculation well. This impression is confirmed by looking at the ratio of the scaled to exact results shown in Figure 6(b): the error introduced by using the scaled approximation is nearly one order of magnitude over the distance range shown here, and increases still further at larger interdonor distances.

\section{D. 'Super-shallow' donors, $\nu=1.1$}

We also show the single-envelope exchange couplings $J_{w}(R)$ in the case of two 'super-shallow' donors. The results are shown in Figure (77). Once again the results are independent of the type of central-cell correction used; furthermore (in the range $R \leq 12 a_{0}^{*}$ ) they are now largely independent of whether the exact Heitler-London approach or the 'Koiller method' is used .

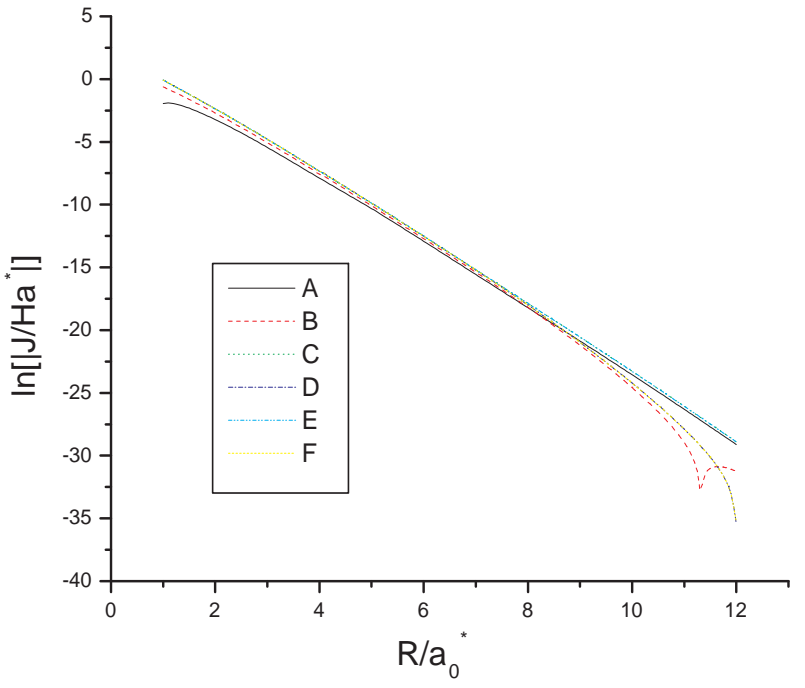

FIG. 5: (Colour online.) The exchange coupling between two deep donor electrons $(\nu=0.7)$ as a function of inter-donor distance $R$. Black solid curve (A): exact Heitler-London method without central cell correction; red dashed curve (B): Koiller's method without central cell correction; green dotted curve $(\mathbf{C})$ : exact Heitler-London method with $\delta$-potential central cell correction; blue dashed-dotted curve (D): Koiller's method with $\delta$-potential central cell correction; cyan dasheddotted-dotted curve (E): exact Heitler-London method with square-well central cell correction; yellow short-dashed curve $(\mathbf{F})$ : Koiller's method with square-well central cell correction. ( $\mathbf{D}$ and $\mathbf{F}$ are indistinguishable at long range). The cusp in curve $\mathbf{B}$, corresponding to a sign change at $R \approx 11 a_{0}^{*}$ is unphysical (see text).

Comparing the results to the hydrogenic case (Figure 44), the dominant difference is once again the change in the exponent. As previously, we compare with the scaled hydrogenic Heitler-London result. Figure 8(a) shows that the matching is much closer than for the deep donors; the relative error is shown in Figure 8(b). In contrast to the deep-donor case, the scaled hydrogenic wavefunction overestimates exchange interaction at short range, but underestimates it at long range. It is perhaps not surprising that the error is smaller in this case, since $\nu$ is closer to the hydrogenic value $\nu=1$ and so the scaling has a smaller effect.

\section{E. Inter-valley effects}

Having calculated $J_{w}(R)$, we include the inter-valley effects by using equation (27). We have calculated the exchange between typical deep donors $(\nu=0.7)$, shallow donors $(\nu=1.0)$, and super-shallow donors $(\nu=1.1)$ in silicon in the case where the donor pair is along a 


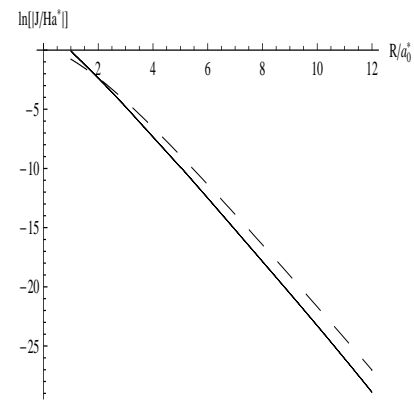

(a)

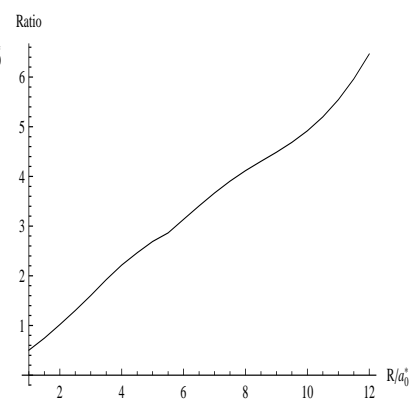

(b)
FIG. 6: Comparison of exact Heitler-London exchange for deep donors $(\nu=0.7)$ with scaled hydrogenic results. (a) Exact Heitler-London exchange $J_{\text {exact }}$ based on $R_{0.7}^{G}$ with a square-well potential central-cell correction (solid line) and scaled exchange splitting using the hydrogenic Heitler-London formula $J_{\text {scaled }}=J_{\mathrm{H}-\mathrm{L} \text {, hydrogenic }}(R / 0.7)$ (dashed line). (b) The ratio $\frac{J_{\text {scaled }}}{J_{\text {exact }}}$ as a function of inter-donor distance.

$\langle 100\rangle$ direction from $1 a_{0}^{*}$ to $12 a_{0}^{*}$ and both donors are in the ground state $\left(A_{1}\right.$ symmetry, so equation (2) applies). We see the exchange interactions still decay exponentially over the whole range, but this decay is mixed with oscillations due to the inter-valley terms as shown in Figure (9).

The solid points in the figure denote the distances corresponding to the cubic lattice constant in $\mathrm{Si}$ (i.e., the actual separations of substitutional sites along [100]). For all three defect types, the interference terms produce deviations from site to site of approximately one order of magnitude, in agreement with the results for hydrogenic defects [13].

\section{F. Distributions of exchange couplings}

In the interpretation of experiments on the ensemble of interacting spins, it is frequently the probability distribution of the nearest-neighbour exchange couplings which is the quantity of most relevance [1, 32]. In order to show how this depends on the donor type, we have used a continuous distribution [33] of donor positions at two different densities, chosen so that the mean nearestneighbour separations are respectively $3.5 a_{0}^{*} \approx 6.8 \mathrm{~nm}$ and $7.0 a_{0}^{*} \approx 13.5 \mathrm{~nm}$, and plotted the distributions of $\log J$ for the three different types of defects in Figure (10). (The lower limit plotted corresponds approximately to the values of $J$ where dipolar interactions begin to dominate over exchange.) All the distributions are extremely broad (as would be expected from the exponential scaling of the exchange with separation) but the strong dependence on the type of donor is evident.

This distribution is important for the choice of donor concentration in samples for applications in quantum information processing; for example, in the scheme proposed in [3] the density should ideally be sufficiently low

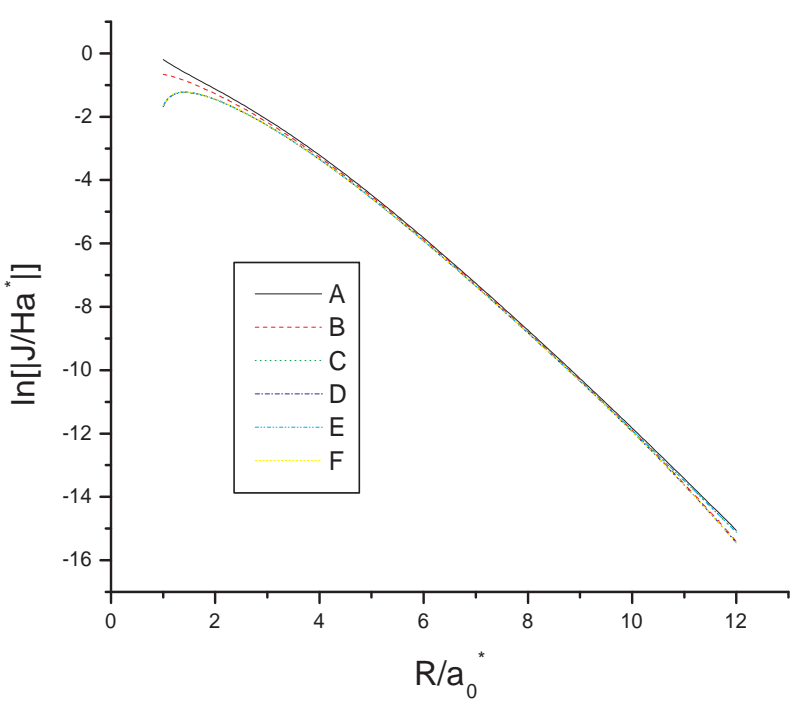

FIG. 7: (Colour online.) The exchange coupling between two super-shallow donor electrons $(\nu=1.1)$ as a function of inter-donor distance $R$. Black solid curve (A): exact HeitlerLondon method without central cell correction; red dashed curve (B): Koiller's method without central cell correction; green dotted curve $(\mathbf{C})$ : exact Heitler-London method based on with $\delta$-potential central cell correction; blue dashed-dotted curve $(\mathbf{D})$ : Koiller's method with $\delta$-potential central cell correction; cyan dashed-dotted-dotted curve (E): exact HeitlerLondon method with square-well central cell correction; yellow short-dashed curve (F): Koiller's method with square-well central cell correction. Notice that these six curves have very similar behaviour over the whole range except at short distance where there is a dependence on the central cell corrections.

that typical exchange interactions between neighbours produce a small evolution of the system on the timescales of gate operation. For deep donors with $\nu=0.7$, typical nearest-neighbour interactions at the lower density are $10^{-4} \mathrm{Ha}^{*}$ or smaller, constraining gate operation times to be at most $0.1 \mathrm{~ns}$.

\section{CONCLUSION}

We have shown that a combination of quantum-defect theory (where Whittaker functions are used to describe simply the long-range part of donor wavefunctions) with model central-cell corrections can be used to describe the electronic structure of donors in semiconductors having binding energies significantly different from the ideal hydrogenic effective-mass value, including in particular the important case of deep donors. From these wavefunctions we have shown that it is possible to calculate the exchange interactions between donors, both in the single envelope-function approximation and by including the 


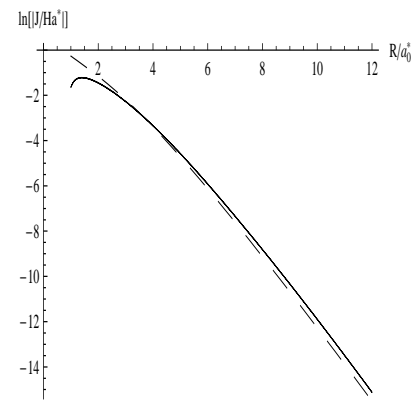

(a)

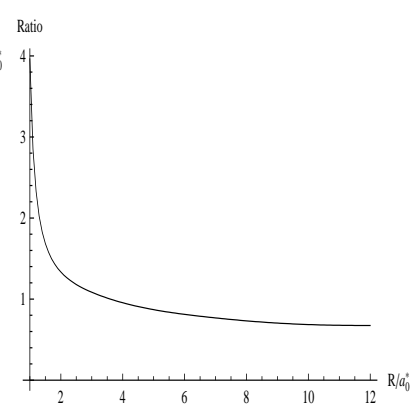

(b)

FIG. 8: Comparison of exact Heitler-London exchange for super-shallow donors $(\nu=1.1)$ with scaled hydrogenic results. (a) Exact Heitler-London exchange $J_{\text {exact }}$ with a square-well potential central-cell correction (solid curve) and scaled exchange splitting using the hydrogenic Heitler-London formula $J_{\text {scaled }}=J_{\mathrm{H}-\mathrm{L} \text {, hydrogenic }}(R / 1.1)$ (dashed curve). (b) The ratio $\frac{J_{\text {scaled }}}{J_{\text {exact }}}$ as a function of inter-donor distance.

interference effects between contributions from different conduction-band minima. These interference effects typically cause the exchange to fluctuate by approximately one order of magnitude between the separations of successive substitutional sites, in agreement with previous calculations for shallow donors.

From the comparison between the exchange calculations performed with and without different central cell corrections, we can see that as expected the correct long-range behaviour of the wavefunction is more important than the form near the nucleus, except when the inter-donor distance becomes very small-however, the Heitler-London like approach we use for exchange is not expected to be accurate at short range. At long range, the central-cell corrections have only small effects on the exchange, although these may be enhanced by the interference of inter-valley terms. However, the inclusion of central-cell corrections is important to obtain consistent results for the exchange between the exact HeitlerLondon approach (equation (21)) and the frequently-used simplification given by equation (14).

Our calculations rely on a Gaussian fit to the true form of the radial function, which is least accurate very near the nucleus and at large distances. At large donor separations we expect the error due to corrections near the nucleus to scale as $\mathrm{O}\left(a^{3}\right)$, where $a$ is the starting radius for the fitting. In our calculations $a=0.01 a_{0}^{*}$, much less than the donor separations $1-12 a_{0}^{*}$ that we consider, so we expect this error to be negligible. The longrange error in the fit is important only at very large donor separations where the spin-spin interaction is no longer exchange-dominated.

We have shown that the type of defect has a significant effect on the distribution of nearest-neighbour exchange couplings experienced at a given density. To summarize the magnitude of the effect, at at separation of $3.5 a_{0}^{*} \approx$ $6.8 \mathrm{~nm}$ the exchange is approximately $0.15 \mathrm{meV}$ for typ-

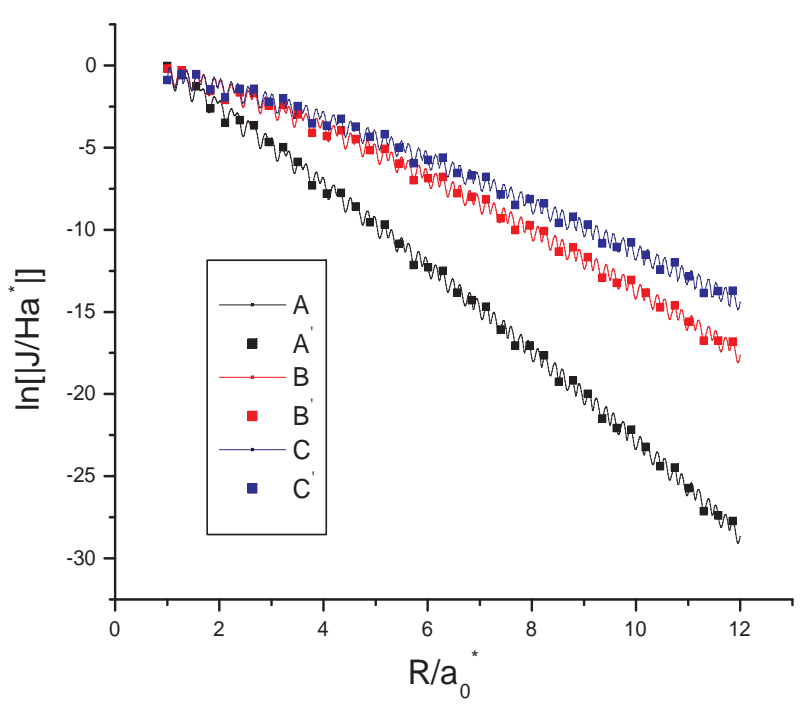

FIG. 9: (Colour online.) Donor-pair exchange including intervalley effects for pair separations along the $\{100\}$ direction as a function of donor distance $\mathrm{R}$. The exchange at integer multiples of the lattice constant is represented by square points. A square-well central-cell correction and the exact HeitlerLondon approach were used for all the calculations. Lower (black) group $\left(\mathbf{A}, \mathbf{A}^{\prime}\right)$ : two deep donors with $\nu=0.7$; middle (red) group $\left(\mathbf{B}, \mathbf{B}^{\prime}\right)$ : two typical shallow donors with $\nu=1.0$; the upper (blue) group $\left(\mathbf{C}, \mathbf{C}^{\prime}\right)$ : two super-shallow donors with $\nu=1.1$.

ical deep donors, $3 \mathrm{meV}$ for hydrogenic shallow donors, and $5 \mathrm{meV}$ for the so-called 'super-shallow' donors. This emphasizes the usefulness of deep donors for the shortterm storage of quantum information, and confirms them as candidates for quantum information processing provided that entangling interactions between them can be switched sufficiently quickly $[2,3]$.

We have also shown that simply scaling the hydrogenic exchange interaction from the Heitler-London formula does not agree quantitatively with our explicit calculations, though it does give the correct order of magnitude for the exchange. The error is most serious for the important case of deep donors.

We believe the methods we present here are quantitatively reliable, and simple enough to provide a useful tool for calculating exchange interactions between donor pairs having arbitrary binding energies.

\section{Acknowledgments}

WW was supported by the Research Councils Basic Technology programme under grant GR/S23506/01. We thank Thornton Greenland, Tony Harker, Andy Kerrdidge, Marshall Stoneham and Dan Wheatley for helpful 


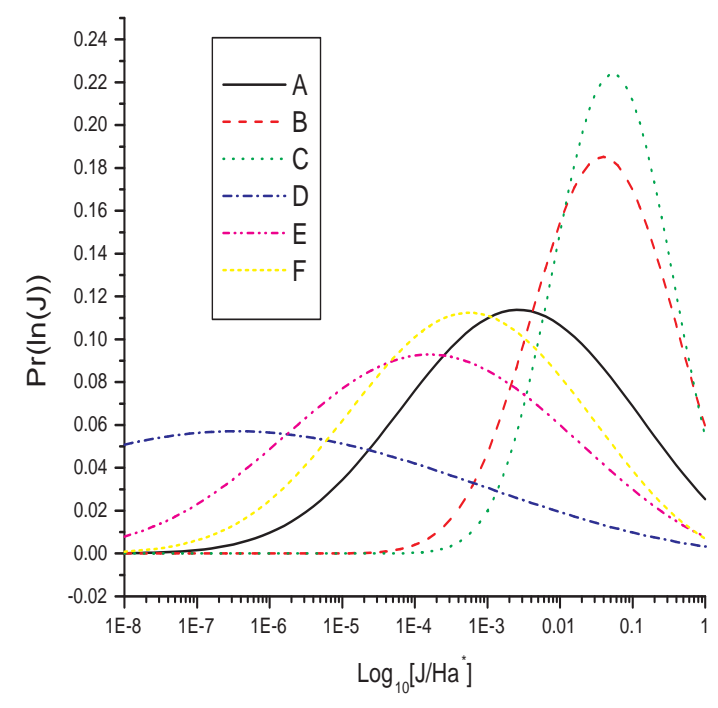

FIG. 10: (Colour online.) The probability distribution of $\log J$ as a function of $J$ (logarithmic scale) for donors with three different binding energies at densities $0.00396\left(a_{0}^{*}\right)^{-3}=$ $5.42 \times 10^{17} \mathrm{~cm}^{-3}$ (curves A, B, C) and $0.0005\left(a_{0}^{*}\right)^{-3}=$ $6.85 \times 10^{16} \mathrm{~cm}^{-3}$ (curves D, E, F). Solid (black) curve $\mathbf{A}$ and dashed-dotted (blue) curve $\mathbf{D}$ : deep donor $\nu=0.7$; dashed (red) curve $\mathbf{B}$ and dashed-dotted-dotted (magenta) curve $\mathbf{E}$ : shallow donor $\nu=1.0$; dotted (green) curve $\mathbf{C}$ and shortdashed (yellow) curve $\mathbf{F}$ : super-shallow donor $\nu=1.1$.

discussions.

[1] K. Andres, R. N. Bhatt, P. Goalwin, et al., Phys. Rev. B 24, 244 (1981).

[2] B. Kane, Nature 393, 133 (1998).

[3] A. M. Stoneham, A. J. Fisher and P. T. Greenland, J.Phys.: Condensed Matter 15, L447 (2003).

[4] J. M. Luttinger and W. Kohn, Phys. Rev. 97, 869 (1955).

[5] C. Kittel and A. H. Mitchell, Phys. Rev. 96, 1488 (1954).

[6] W. Kohn, J. M. Luttinger, Phys. Rev. 98, 915 (1955).

[7] J. C. Slater, Quantum theory of molecules and solids, McGraw-Hill Book Co., New York (1963).

[8] C. Herring, Rev. Mod. Phys. 34631 (1962).

[9] C. Herring and M. Flicker, Phys. Rev. 134, A632 (1962).

[10] A. Miller and E. Abrahams, Phys. Rev. 120, 745 (1960).

[11] R.C. Enck and A. Honig, Phys. Rev. 1771182 (1969).

[12] P. R. Cullis and J. R. Marko, Phys. Rev. B 1, 632 (1970).

[13] B. Koiller, et al., Phys. Rev. Lett. 88, 027903 (2001).

[14] W. Heisenberg, Z. Phys. 49, 619 (1928).

[15] B. Koiller, et al., Phys. Rev. B 66, 115201 (2002).

[16] C. J. Wellard, L. C. L. Hollenberg, F. Parisoli, et al., Phys. Rev. B 68, 195209 (2003).

[17] B. Koiller, R. B. Capaz, Xuedong Hu, et al., Phys. Rev. B 70, 115207 (2004).

[18] A. K. Ramdas and S. Rodriguez, Rep. Prog. Phys. 44, 1297 (1981).

[19] T. G. Castner, Jr., Phys. Rev. 130, 58 (1963).
[20] H. Barry Bebb, J. Phys. Chem. Solids 28, 2087 (1967).

[21] M. J. Seaton, Rep. Prog. Phys. 46, 167 (1983).

[22] A.M. Stoneham Theory of Defects in Solids $§ 23.4$, Oxford (1975).

[23] M. Abramowitz and I.A. Stegun, Handbook of Mathematical Functions, Dover Publications (1965).

[24] H. Nara and A. Morita, J. Phys. Soc. Jpn. 21, 1852 (1966).

[25] H. Nara and A. Morita, J. Phys. Soc. Jpn. 23, 831 (1967).

[26] A. Kerridge, S. Savory, A. H. Harker and A. M. Stoneham, J. Phys.: Condens. Mat. 18, S767-S776 (2006).

[27] S.T. Pantelides and C.T. Shah, Phys. Rev. B 10638 (1974).

[28] H. M. James and A. S. Coolidge, J. Chem. Phys. 1, 825 (1933)

[29] W. Kolos and N. Wolonectz, J. Chem. Phys. 43, 2429 (1965).

[30] L. P. Gor'kov, L.P. Pitaevski, Dokl. Akad. Nauk. SSSR 151, 822 (1963).[English Transl.: Soviet Phys.--Dokl. 8, 788 (1964).]

[31] S. F. Boys, Proc. Roy. Soc. A 200, 542 (1950).

[32] R.N. Bhatt and P.A. Lee, Phys. Rev. Lett. 48, 344 (1982).

[33] S. Chandrasekhar, Rev. Mod. Phys. 15, 1 (1943). 